\title{
CANNOT PSYCHOLOGY DISPENSE WITH CONSCIOUSNESS?
}

\author{
BY ELIOTT PARK FROST \\ Yale Unioersity
}

In his essay: 'Does consciousness exist?', posthumously published, William James writes: "For twenty years past I have mistrusted consciousness as an entity; for seven or eight years past $I$ have suggested its non-existence to my students. ... It seems to me that the hour is ripe for it to be openly and universally discarded."

Such a pronouncement is welcomed, von Anfang an, by the Realists and Radical Empiricists in philosophy, who accept the term 'consciousness,' as name for a function of the thinking process. Such a pronouncement is equally welcome to the students of animal behavior, who read in it, however, only a confirmation of what they have long proclaimed: the nonessentiality of the concept of consciousness. Strangely enough, it is in psychology itself that a readjustment in extrinsic terms proves difficult. The self-consistent textbook of a psychology, thus objectively viewed, has yet to appear. If one is ever to be written, it can neither ignore what is commonly termed 'conscious process'; nor may it still use the psychic terminology uncorrected.

The business of our discipline is to describe, and to explain, certain phenomena. Description heretofore, has meant to talk in mental terms: of sensations; of percepts as synthesized sensations; of memory, of imagination, and the like. Explanation heretofore has meant to give the physiological correlate of these psychic processes: to speak of neurones, synapses, and final common paths. Whatever nice distinctions the instructor may make for himself, it is certain that the average student leaves his first year in psychology with the notion of two very clear-cut entities: a physical process that one can tap, detect, 
measure; and a further somehow-psychic process, part experienced through introspection, part conceived of.

At the outset I show my hand. It is my personal bias, that, as data for a course in elementary psychology at least, psychic terminology with its present connotations, is confusing. I deplore the awkward straddle in which psychology is at present. We are trying to ride two horses at once, the horse of philosophy and the horse of behavior, and they are proving as mutually irreconcilable as the horses in the Platonic figure, one horse being white, and the other horse black. Let us keep to one mount or the other with some consistency.

"Whoever blots out the notion of consciousness from his list of first principles," must indeed as James says, "still provide in some way for that function's being carried on." It is my present, perhaps over-ambitious intention, to attempt an explanation of the "fact that things not only are, but (that) they get reported, are known," in a strictly physiological way, without any conscious or psychic implication whatsoever.

The term 'awareness,' emphasized by James himself, and reimpressed upon students in every psychological class-room, has always a mental, rather than a physiological connotation. To be aware is to be conscious. To prove awareness is to prove the presence of consciousness. Once establish the processes of awareness in an organism, and one demonstrates the presence of consciousness in that organism.

I beg that for a few moments, however, you will agree with me to understand the term 'awareness' in a different sense: namely, that it shall have exclusively a physiological import. So that, if you grant a friendly ear to this proposal, we shall speak of a physiological mechanism as, at times, becoming aware. And we shall test the presence of such awareness on the part of such a mechanism according to the usual criterion of behavior: does the given stimulus elicit from it a response. If, from a nervous structure, a stimulus does elicit any sort of response, in so far this shall sufficiently justify the assertion: "the organism is become aware of that stimulus." If, on the contrary, the nervous structure, upon irritation, makes no response of any nature, it must be then maintained: "the 
organism is unaware," or, to use the synonym, is 'insensible.' And we shall remember that all the while the usual mental or psychic connotation of these terms is to be rigidly excluded.

With this distinction as a basis, let us investigate a simple type of behavior.

Within a dark room suddenly flash upon a nervously sensitive visual end-organ, a beam of light. If the pupillary reflex take place, as will transpire in the normal eye, it may then be affirmed that the eye-mechanism has become aware of the stimulus; and the resultant behavior, namely, the closing of the eye, is proof positive that such is the case. Such a primary reflex may occur, of course, wholly independent of any functioning on the part of higher neural centres.

Let us designate such a simple sensori-motor path as that involved in nervous response to a stimulus, as an Alpha-arc. The term alpha-arc shall then characterize any simple, single, sensori-motor path, initiated by some peripheral stimulus, and resultant in some end-effect. For an alpha-arc to function it is merely necessary that stimulus elicit nervous response.

But an alpha-arc may involve a longer circuit. If, in the dark room, the tiny flash come now from the side, it will naturally be met by a turning of the eye-ball, to bring the stimulus within the direct line of regard. Beyond structures involved in the previous illustration, there are now included at least the thalami, and the optic lobes. Still, however, we shall speak as before, shall we not, of a sensitive mechanism that has become aware of a stimulus, and has responded to that stimulus by adaptive behavior? We still have an arc, have we not, that may run its course independent of any so-called 'conscious coefficient,' and still result nevertheless, in accommodative behavior? In the lower organisms we call such behavior tropic; in human behavior we speak of it as completely habitualized or instinctive reponse.

As a matter of fact, however, whenever an alpha-arc functions so as to include the specific cortical structures, for instance, the temporal or optic lobes, we presume that there is always the further likelihood that the great complication-areas, or association areas of the cortex, be stimulated as well. When 
such a further arc, aroused by an alpha-arc rather than by a peripheral stimulus, is set in function, let us denote it as a Beta-arc. In brief, then, when a stimulus falls upon a sensitive neural mechanism, it will normally arouse an alpha-arc, and this alpha-arc may in turn arouse a beta-arc; the latter being alone provoked by the alpha-arc, and presumably having for its seat the association areas of the cortex. The only experimental criterion of such a beta-arc will of course, again be behavior; but behavior significantly different from the response of the simple alpha-arc. Just what this modified type of betaarc response involves can be shown in a moment.

In the case now before us, we speak of the beta-arc as becoming aware of an alpha-arc, in precisely the same sense that we spoke a moment ago of the alpha-arc as aware of the external stimulus, and for the same reason: it responds to stimulation, characteristically. The alpha-arc is unaware of itself, but aware of the external stimulus; the beta-arc is unaware of itself, but is aware of the alpha-arc. Only when and if the beta-arc in turn arouse some still subsequent arc, as a gammaarc, can it itself become an object of awareness.

Once again, then, all simple sensori-motor arcs having their loci in the first and second levels, to use MacDougall's phrasing; aroused by stimuli peripheral to the central nervous system, and eventuating in behavior, we shall call alpha-arcs. And all arcs which are stimulated alone by alpha-arcs, having their neural seat in the association areas, and related only through intermediate structures to the end-organs, but still eventuating in characteristic behavior-these we shall call beta-arcs. Neither an alpha-arc nor a beta-arc is then aware of itself, but the beta-arc becomes aware of the alpha-arc; the alpha-arc becomes aware of the external irritating object. In a word, no arc is self-sensing, but any arc may become object for a succeeding arc. And what holds of an individual arc, holds equally true of complexes or nexuses of such arcs.

By introspection, psychology alleges that one holds for a moment as object of an awareness-process, a just-previous process occurring within one's own private nervous system. But such a prior process is commonly dubbed 'mental,' or 
'psychic,' and the assertion is made that while such a process is unaware of itself, it reflects upon another mental process, contiguous, but just prior. Even for the introspectionist, that is, no process can become aware of itself. All introspection involves reflection, temporal succession. For the introspectionist there must be two processes, both alleged psychic in nature, where one process takes for its object another process, just prior.

In accordance with the tenets of ordinary psychology, then, if consciousness were cinematographic, instead of continuous, mental life would be impracticable; for while the integrity of the first process would remain unimpaired, this process could not become aware of itself, nor by hypothesis would it remain as object for a succeeding process, and hence there would exist the possibility of no awareness-experience whatsoever, whether dubbed 'conscious' or physiological. In short, if there were no memory; if no content cast its influence beyond the fleeting instant, and life were but a succession of momentary incandescences, each swallowed up in oblivion so soon as completed, then reflection, and with it introspection, would become out of the question.

Well! If no process can experience itself, be within itself both subject and object, what grounds exist for labelling any such process, 'psychic'? Is not the ascription to such processes of a psychic content, a wholly gratuitous performance? Are not the mutual relations of these arcs to be understood in simpler terms?

This the present writer believes. For him the term 'consciousness' with its psychic implicates, has long seemed to be a misnomer. Within his so-called 'world of experience,' he can find no psychic attachment. The whole conception of a mental, extra-physiological experience appears to him only as a pleasant speculation for the philosopher to play with; but one that becomes useless, if not misleading, for the teaching of psychology as a science. Is not behavior adequately explained as the resultant, in our terminology, either as the functioning of an alpha-, or as the functioning of a beta-arc, or group of such arcs? Does not this cover all that is now meant by 'conscious process'? 
If we yield (perhaps to the philosopher), all 'life-rights' in the term 'consciousness,' on the ground that it is a misleading concept, we can then speak of an alpha-arc as an awarenessprocess, having for its biological goal the immediate adjustment of organism to stimulus. In definition of the further beta-arc process, the present writer has elsewhere suggested the term: 'consciousizing process.' The biological significance of this latter lies in its ability to take as an object (i. e., to be stimulated by), prior awareness-processes, or cortical retentions of such processes (commonly called images), or both. In this way the immediate afferent impulses (alpha-arcs) are influenced by previous organic experience, and resultant behavior is correspondingly modified. In the simple awareness-processes, the nervous impulse has a fatal course to run, passing from the point of stimulation in the end-organ directly to the musculature over hereditary or canalized paths. But if the alphaimpulses in passing, set off further beta-arcs, facilitation or inhibition of one sort or another takes place, and the final behavior is modified, since there is now involved elaboration of the original impulse in terms of the past experience of the particular nervous system implicated.

There is no reason to suppose that nervous impulses differ in kind. An alpha-arc and a beta-arc differ only in that they have unlike objects. In the one case the object is a peripheral stimulus; in the other case it is a central one. If one prefer to call this awareness, on the part of one nerve path, of another nerve path, 'conscious awareness,' to distinguish it from the simple reflex type of awareness, there can be only the objection that the majority of present-day students of our discipline will feel that somehow something psychic has crept in; a conception, from our point of view, both useless and disconcerting. If we can but purify our psychological concepts of their mysterious connotations, there may be advantage in continuing to use the old-time nomenclature.

Alpha-arc awareness is after all unlike beta-arc awareness, as a neural experience. In speaking of the latter we might still say: 'sensation;' 'image;' 'thought;' 'mental process.' So far as these everyday terms express only a unique physio- 
logical experience, different from a mere awareness experience, there is no excuse for replacing them by neologisms. But sensations will no longer now be 'first things in the way of consciousness,' but the second. There must always be at least two physiological processes, successive in time, for us with propriety to call one of them a consciousizing process, or sensation. The iris, responding to a light stimulus, can never get a sensation.

At just what point in biological evolution, beta-arcs first evolved to supplement the simple alpha-arcs, is no doubt largely a matter of speculation. They would seem to be called for, however, when the reflexes prove no longer adequate to cope with the environment, except by reference to past experience.

Past experience, retained as cortical disposition, may of course become the object of beta-arc awareness, no less than a fresh afferent path; so that the responses sent to the final common paths, to use Sherrington's terms, are the resultants of alpha-arcs, recent and remote, plus the effect of consciousizing processes upon them both.

If we are to speak of a beta-arc in function, as a 'consciousizing process,' we may speak of an alpha-arc as a 'preconsciousizing process,' or at other times, as a 'consciousized process.' The former term would characterize those alpha-arcs that are independent of beta-arcs, being originally autonomous, viz., the reflexes. And the latter term would characterize those alpha-arcs which no longer arouse beta-arcs, although they formerly may have done so, viz., habitual mechanisms. These exhibit the effects of beta-arc action, but no longer normally engender it.

Activity of an alpha-arc gives the organism, say, red-awareness. This means that the retina has responded to an ether vibration of $44^{\circ} \mu \mu$ in specific fashion: a different response from that which it makes to vibrations of, say, $790 \mu \mu$. If this arc now an instant later arouses a consciousizing process (beta-arc) we get what we. may call the 'sensation red.' Can either introspection or logic demand any further description or explanation of this 'sensation-red-experience' than to say that a 
nervous impulse has passed through the cortex, and there aroused a second impulse which takes it as an object of awareness? Does the postulation of any psychic entity clarify or add to the data thus offered by physiology?

The 'intimate warmth' of which James speaks, does not present us with a Self. The 'warmth', indeed, is explicable: the object of regard in such cases is a fresh neural path, just now in function. But to become aware of the warmth requires still a separate, subsequent, neural path, as yet unaware of itself.

So ingrained is the 'common-sense view' that physiological processes are the vehicle only of psychic processes which play upon and through them, that it is difficult at first to comprehend how a physiological process in function is itself consciousness at the moment, completely described; and that any further implication is unwarranted. But cannot psychology approach nearer to the scientific ideal of all disciplines by considering the terms 'consciousness' and 'mind' merely as handy ways for expressing the reactions or awarenesses on the part of processes beta, gamma, delta, and so on; of processes alpha, beta, and gamma, respectively just prior? Is not what is commonly meant by 'consciousness,' so far as psychology is concerned, more nearly described as a 'beta-arc functioning,' than to speak, either of 'an elementary psychic process,' or even of 'a knowing function'? 